\title{
MONITORING SERVICE "HEALTH" IN INTELLIGENT, TRANSPARENT OPTICAL NETWORKS
}

\author{
Carolina Pinart ${ }^{1}$, Abdelhafid Amrani ${ }^{1}$, and Gabriel Junyent ${ }^{1,2}$ \\ ${ }^{\prime}$ Centre Tecnològic de Telecomunicacions de Catalunya, c/ Gran Capità 2-4 08034 \\ Barcelona,e-mail: \{carolina.pinart, abdelhafid.amrani\}@cttc.es \\ ${ }^{2}$ Universitat Politècnica de Catalunya, c/ Jordi Girona 1-3 D4 08034 Barcelona, e-mail: \\ junyent@tsc.upc.es
}

\begin{abstract}
This paper focuses on the design and implementation of a low-complexity dialogue mechanism between the management and transport planes of an alloptical network. This dialogue aims at exchanging relevant information from monitoring the performance and degradation of optical signals with minimal disturbance to the optical services and minimum knowledge of the transport history of data, with a view to ensure service quality. Complexity of the dialogue is measured in terms of response delays in the event of failures.
\end{abstract}

\section{INTRODUCTION}

The accelerating growth of data traffic is motivating the research for more efficient, flexible, intelligent optical network architectures. Optical networks promise to be the underlying next generation technology for the future Internet and broadband networks, being Internet Protocol (IP) over Wavelength Division Multiplexing (WDM), IP/WDM, one of the most promising candidates.

Performance monitoring plays a fundamental role in the deployment and future evolution of the optical network industry, because assuring service quality (QoS) will be key for the success of next-generation networks, which are to enable dynamic, differentiated optical services. Therefore, efforts are underway to investigate new ways to monitor the performance of data on an optical network with minimal conversion to electronics and minimal disturbance to the signal on 
the fiber, as well as to integrate fiber-optic networks, communications and signal processing, and optical network protocols.

As for protocols in IP/WDM, Generalized Multi-Protocol Label Switching (GMPLS) is thought to be an integral part of next-generation optical networks, especially as control plane of the Automatic Switched Optical Network (ASON) [1], because it renders optical networks intelligent. Concerning the management information protocol, the Simple Network Management Protocol (SNMP) [7] has been the industry reference for network management since the last 1980s; SNMP agents are installed in almost any system to enable remote access to its components, making SNMP a de facto standard for networked hardware management [6].

This paper focuses on the mechanisms for Optical Performance Monitoring (OPM) in an IP/WDM network, especially the dialogue of the transport and management planes to exchange relevant information obtained from monitoring the performance and degradation of signals with minimal disturbance to the signals and minimum knowledge of the transport history of the data to assure QoS.

The remainder of this paper is organized as follows. Section 2 is devoted to the reference model and parameters for monitoring the health of optical services, as well as the different inputs for such monitoring. Section 3 describes the experimental ASON/GMPLS testbed, focusing on the data model and the SNMP agent implementation for service monitoring. Section 4 deals with preliminary performance evaluation results of the dialogue. In Section 5 we draw conclusions.

\section{REFERENCE MODEL AND MONITORING PARAMETERS}

The reference model of OPM has three layers. Starting from the WDM input, we encounter channel management, then channel quality and protocol performance [6]. These layers are known as transport, signal quality and protocol monitoring, respectively. In this work, we only deal with transport monitoring due to the fact that the method chosen for implementing OPM is a non-disruptive all-optical dedicated monitor that taps the optical signal on a Dense WDM (DWDM) fiber. In this case, the monitor is shared among the wavelengths carried in the fiber. At this layer, the most commonly suggested monitoring parameters are [6]: aggregate power, channel power, spectral optical signal-to-noise ratio (SNR) and channel wavelength. In contrast, time domain parameters such as Bit Er ror Rate (BER) and Q-factor cannot be obtained by the considered OPM owing to its transparent nature.

In this work, an optical service, understood as the provisioning of an optical channel (wavelength), encompasses the following QoS parameters: establishment 
and release delays [7], which may be monitored directly in the manager, channel "health" (obtained through OPM) and status of optical components (component failures) that might lead to service degradation, such as lasers, receivers and active switching and control components. To retrieve these QoS parameters, several monitoring points must be set in the optical network. Major component failures may take place in the control plane (faults in the GMPLS-based Optical Connection Controllers, $\mathrm{OCC}$ ) and in the transport plane. Therefore, monitoring points for component failures are split in the optical node controller ( $N C$ in Figure 1) and management agents (OCC and all-optical add-drop multiplexer, OADM, illustrated as Agent in Figure 1) at each node. Moreover, the impairments that may affect the optical signal quality, causing undesirable variations of the channel power, frequency or OSNR, are monitored by a transport monitor (Figure 1). Note that degraded BER and restoration delay are usually considered in the event of failures [7], but since we use all-optical monitoring at WDM layer and fast physical protection at Optical Multiplex Section level (OMS), these parameters are not considered in this work.

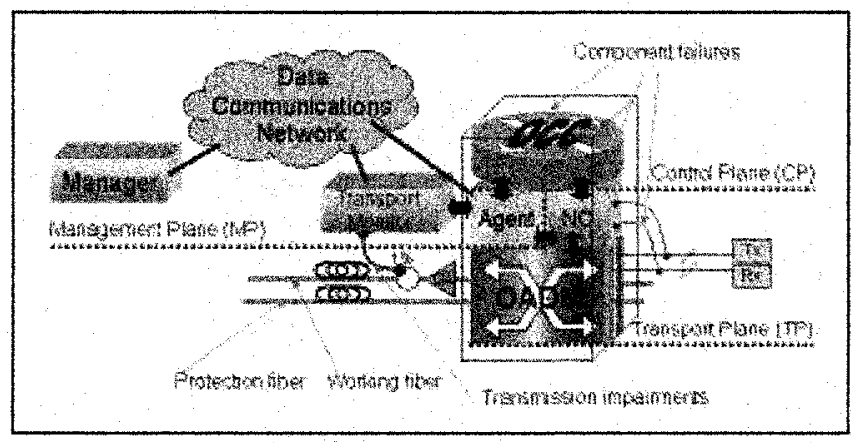

Figure 1. Monitoring in an IP/WDM node

\section{EXPERIMENTAL ASON/GMPLS TESTBED}

The experimental testbed ${ }^{1}$ used in this paper is a DWDM transport network enabled with optical intelligence through a GMPLS control plane to allow realtime, dynamic configuration of optical services between multiple clients (ASON). Due to economic reasons, 8 wavelengths per fiber, spaced $100 \mathrm{GHz}$ (ITU channels from 30 to 37 ) at speeds up to $2.5 \mathrm{Gbps}$ are available. Due to its relevance in this

1 ADRENALINE testbed: All-optical Dynamic REliable Network hAndLINg IPEthernet Gigabit traffic with QoS. http://www.cttc.es/adrenaline/ 
work, the transport plane's architecture is summarized in Section 3.1, whereas the overall architecture of the testbed is further detailed in [5].

\subsection{Transport plane}

The testbed's transport plane is an Optical Transport Network (OTN) [2] that provides uni and bidirectional optical channels transparent to the format and payload of client signals. Moreover, it is the source of information about the state of connections and their performance, which is central to OPM. All laser sources are fully tunable, and no wavelength converters are contained in the OADMs [5] (architecture depicted in Figure 2). In this work, the configuration of the OTN is a unidirectional ring with OADMs. So, each link has two unidirectional fibers, one of which is used for OMS protection [2]. In the architecture of an OADM, an incoming signal from the working fiber is demultiplexed into the 8 wavelengths before entering the $2 \times 2$ all-optical switches, each of which may either drop a wavelength (maximum 4 wavelengths dropped at each OADM) and optionally add a new one, or pass through that wavelength. Note that each wavelength has a switch associated, so that all wavelengths may be added/dropped.

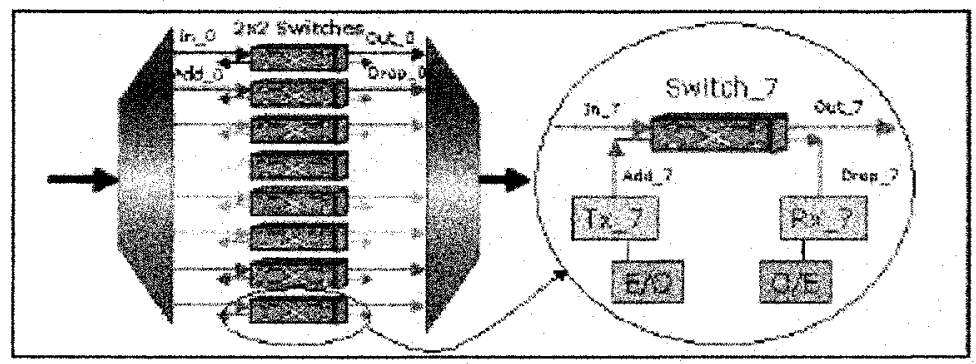

Figure 2. Architecture of an OADM

Once all output wavelengths are ready, they are multiplexed and the DWDM signal is inserted in the outgoing fiber (Figure 2). At this stage, $1 \%$ of optical power of the DWDM signal is tapped and brought to an all-optical signal performance monitor. This equipment allows a first estimation of the optical signal quality by measuring the channel power, frequency, OSNR, and their respective drifts in a totally transparent manner, not only at each optical node output but at the input as well. Consequently, node or link failures can be detected. Moreover, degradation thresholds allow proactive fault management by detecting and avoiding or correcting a failure before it occurs. In contrast, conventional digital methods of optical signal monitoring such as BER test, Bit Interleaved Parity (BIP) check or Cyclic Redundancy Check error (CRC) enable the detection of a failure in 
an optical network without identifying its origin, or its localization. In addition, the failure detection is only at the electrical edge nodes resulting in a high granularity of the detected fault, higher network resource utilization for restoration and slower recovery. Moreover, digital methods of fault detection are specific to the digital characteristics of the optical signals and generally require optical demultiplexing, optical to electrical conversion and synchronization to the bit rate.

A description of the processing mechanisms for OPM data, as well as of the most relevant network elements for optical service monitoring follows.

\subsection{Optical node controller}

Active components of the transport plane are the $2 \times 2$ optical switches, the transmitters (lasers), the transceivers ( $\mathrm{E} / \mathrm{O}$ and $\mathrm{O} / \mathrm{E}$ ) and the photonic receivers (Figure 2). Moreover, if the OADM is reconfigurable, an additional (active) matrix switch is needed for the distribution stage [5], not considered in this work. Last but not least, optical amplifiers (OA in Figure 1), located in the optical links, are also active. Then, the optical NC is responsible for monitoring the state of these active components, as well as for changing their state according to the needs of the control and management planes, which come mainly from service provisioning [3]. In this work, the testbed's NCs contain a 32-bit micro-processor with RS232 and RJ45 ports. The serial port communicates with the active optical components at a speed of $115,2 \mathrm{Kpbs}$. The NC interacts with these components through a Field Programmable Gate Array (FPGA) circuit that ensures electrical connections both for information exchange and electrical supply to the optical components. The micro-processor is a Linux platform that performs the following:

Proxying. Execution of requests from the OADM agent (Section 3.3) and the manager (through the agent), such as full status information of receiver $\mathrm{Rx}_{-} 7$. Retrieval of transmission information from the agent and/or the optical monitor.

Connection Controller Interface ( $\mathrm{CCI}$ ). Execution of commands from the $\mathrm{OCC}$ of the optical node (control plane) related to a provisioning process. For instance, add a channel with wavelength $1553.33 \mathrm{~nm}$ to output Out_7, which means changing status of laser Tx_7 from OFF to ON, setting the laser channel to 30 , setting the laser variable optical attenuator (VOA), changing the status of the optical $2 \times 2$ switch from bar to cross, and setting the matrix switch input/output.

Monitoring. Periodic retrieval of status information from the optical components. Alarms and notifications in case of failures to the OADM agent. Status information involves the characteristics of the following components:

- Transceivers: input/output power.

- Lasers (Transmitters): laser temperature, optical power, wavelength stability, laser current, laser temperature, aging failure and port failure, in addition to output power from external modulators. 
- Avalanche photodiodes optical receivers (APD): loss of power alarm detection.

\subsection{OADM agent}

ITU-T Recommendations of X.700 Series or M.3010 are examples of conceptual (information) modeling of the management plane, including service provisioning. Such modeling is independent of specific implementation, and defines relationships between managed objects. Since control plane and transport plane ele ments (OCCs and OADMs, respectively) contain management agents (Figure 1), as well as Management Information Bases (MIB) and a message communication function, encompassing a management information protocol, the manager is able to access the information models of both the control and transport planes. Therefore, we focus on a data model for service monitoring. As for the OADM agent's MIB, it contains the IETF module OPT-IF-MIB [4] and the module OHW-CTTC-MIB (Figure 3, right), developed in this work. OPT-IF-MIB defines objects for managing optical interfaces associated with WDM systems or characterized by the OTN architecture. The main object of OHW-CTTC-MIB is opticalHwTable, complementary to OPF-IF-MIB because it contains information of transmitters, receivers and switches, indexed by opticalSwitchNumber. The example on Figure 2 (right) would have all parameters of opticalHwTable indexed by 7 , since the $2 \times 2$ switch is Switch_7.

The OADM management agent (Figure $3 \mathrm{~b}$ ) is based on SNMP (manager-agent paradigm) [8], which has been chosen as management information protocol because it is the industry reference. This agent has four main functions: responding to monitoring information queries, sending alarms/notifications of resource status, proxying with the $\mathrm{NC}$ and the monitor (retrieval of frequency assignment and stability, power level and OSNR for each optical channel, at the input and at the output of each node) and retrieval of power level of optical amplifiers (status information), via SNMP. The transport monitors also respond to SNMP queries made by the manager and send alarms caused by transmission impairments. Instead of overloading the network with status information, messages are only sent when a failure is likely to occur (warning), so that the control and management planes may both react proactively without service disruption, and reactively after a failure (fault). The monitor used in this work is Digital Lightwave's Optical Wavelength Monitor, which is all-optical and monitors power, wavelength (every $10 \mathrm{~ms}$ ) and optical SNR (every $100 \mathrm{~ms}$ ) for multiple channels on DWDM networks. Alarms are sent via SNMP according to the monitor's DIGL-OWM-MIB module. 


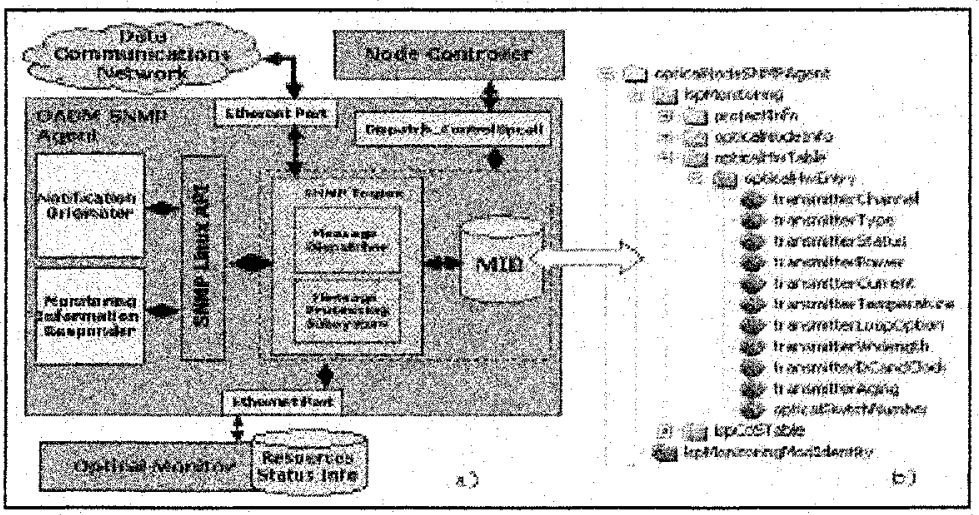

Figure 3. a) OADM architecture and b) OHW-CTTC-MIB module (transmitter objects)

\section{PRELIMINARY PERFORMANCE EVALUATION}

We consider the worst-case failure scenario depicted in Figure 5a. Laser 1, which transmits data of a Premium service [7] in channel 30, experiences a power loss that degrades the Service Level Agreement (SLA). As soon as the NC detects the fault, it informs the OADM agent, which forwards the event to the manager (SNMP Trap). Due to the severity of the fault, the manager requests (SNMP Set) that data be transmitted in the same channel by idle laser 2. The Data Communications Network (DCN) is congested and the distance between the manager and agent is $400 \mathrm{~km}$. Figure $5 \mathrm{~b}$ plots OPM performance in terms of delay.

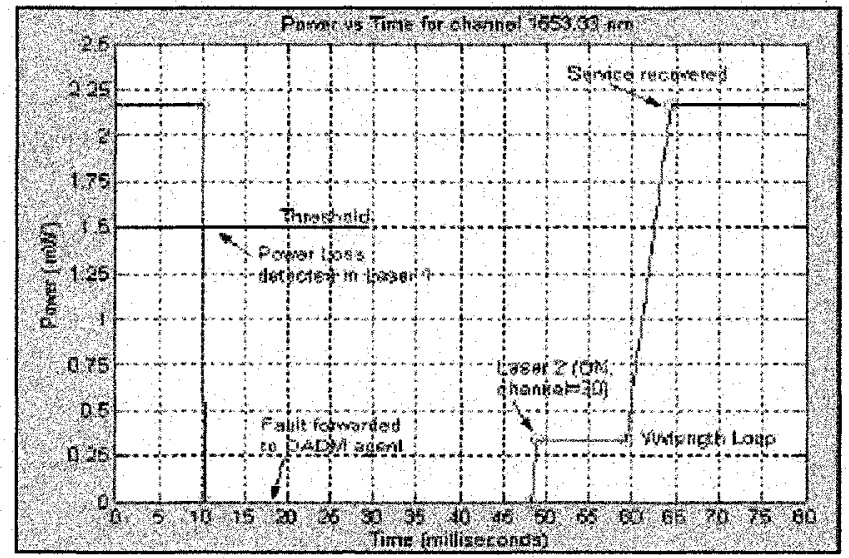

Figure 5. a) Test setup and b) Channel power vs. time (ITU channel 30, congested DCN) 


\section{CONCLUSIONS AND FUTURE WORK}

We have presented implementation issues to achieve a simple dialogue among the optical management plane and the transport and control planes to forward relevant information from monitoring the performance and degradation of signals in an all-optical network with minimal disturbance to the optical services and minimum knowledge of the transport history of data, with a view to ensure service quality. Preliminary results assess simplicity in terms of low delays (worst case is around $50 \mathrm{~ms}$ in heavily congested DCN). As far as we know, recovery times with degraded SLA are not specified in the literature, whereas full recovery times are suggested as $50 \mathrm{~ms}$ for Premium class services [7]. Therefore, future work encompasses optimizing the implementation of the OPM dialogue presented to comply with the most restrictive SLAs that are being proposed.

\section{ACKNOWLEDGMENTS}

This work is part of the NetCat, EMPIRICO (PU-2002-56) and TBONES (ITEA 02024 and FIT-070000-2003-936) research projects.

\section{REFERENCES}

[1] ITU-T G.8080, Architecture for the Automatically Switched Optical Network (ASON), November 2001.

[2] ITU-T G.872, Architecture of optical transport networks, November 2001.

[3] Pinart, C.; Junyent, G.; On implementing a management plane for service provisioning in IP over reconfigurable WDM networks, in Proceedings ONDM 2004, pp. 465-480.

[4] Lam, H.-K.; Stewart, M.; Huynh, A.; Definitions of managed objects for the optical interface type, IETF RFC 3591, September 2003.

[5] Muñoz, R.; Pinart, C.; Martinez, R.; Amrani, A.; Junyent, G.; An experimental ASON based on OADM rings and a GMPLS control plane, Journal of Fiber and Integrated Optics, Vol. 23, Nb. 2-3, pp. 67-84, March-June 2004.

[6] Kilper, D. C., et al. ; Optical Performance Monitoring, IEEE/OSA Journal of Lightwave Technology, Vol. 22 (1), pp. 294-304, January 2004.

[7] Fawaz, W., et al; Service Level Agreement and provisioning in optical networks, IEEE Communications Magazine, Vol. 42 (1), pp. 36-43, January 2004.

[8] Harrington, D. Ed.; An architecture for describing SNMP management frameworks, IETF RFC 2571, April 1999.

[9] Muñoz, R.; Pinart, C.; Martínez, R.; Sorribes, J.; Junyent, G.; Experimental demonstration of two new GMPLS lightpath setup proposals for soft-permanent connections over a unidirectional OADM ring implemented on EMPIRICO testbed, in Proceedings III Workshop MPLS Networks, pp. 79-95. Girona, March 25 -26 2004. 